Zbl Arbeitsmed 2021 $\cdot 71: 86-96$ https://doi.org/10.1007/s40664-020-00414-6 Eingegangen: 14. Januar 2020

Überarbeitet: 4. November 2020

Angenommen: 7. November 2020

Online publiziert: 10. Dezember 2020

(c) Der/die Autor(en) 2020

\section{Hintergrund und Fragestellung}

Eine Ursache für Arbeitsunfälle und arbeitsbedingte Erkrankungen sind in der Regel unzureichende sicherheitsbezogene Wissensgrundlagen und Fähigkeiten der Beschäftigten. Studien haben gezeigt, dass der wesentliche Prädiktor für die Sicherheits-Compliance einer Person $[5,10,14]$ arbeitsschutzbezogenes Wissen ist [2]. Um im Rahmen der Arbeitsaufgaben Gefahrensituationen $\mathrm{zu}$ identifizieren und geeignete Maßnahmen zur wirksamen Vermeidung oder Minimierung der Gefährdungen abzuleiten, muss vom Beschäftigten der grundlegende Zusammenhang zur Gefährdungsentstehung verstanden, abstrahiert und auf andere Gefährdungssituationen übertragen werden. Dabei zeichnen sich Gefährdungssituationen durch eine hohe Komplexität aus, der Abruf von bekannten Handlungsabläufen ist daher in den seltensten Fällen ausreichend. Stattdessen bedarf es einer soliden Wissensgrundlage und klarer Problemlösestrategien, die adaptiv sowie gefährdungsbezogen angewendet werden können.

Die Vermittlung sicherheitsbezogener Kompetenzen und sicherheitsbezogenen Wissens als Voraussetzung kompetenten Handelns ist ein Ziel in der beruflichen Ausbildung. Sowohl in den Ausbildungsverordnungen als auch in den KMK-Rah-

\author{
Anke Kahl ${ }^{1} \cdot$ Carolin Frank ${ }^{2} \cdot$ Ralf Erlebach ${ }^{2}$ \\ ${ }^{1}$ Lehrstuhl für Sicherheitstechnik/Arbeitssicherheit, Fakultät Maschinenbau/Sicherheitstechnik, Bergische \\ Universität Wuppertal, Wuppertal, Deutschland \\ ${ }^{2}$ Lehrstuhl für Didaktik der Technik, Fakultät Maschinenbau/Sicherheitstechnik, Bergische Universität \\ Wuppertal, Wuppertal, Deutschland
}

\title{
Eine kritische Untersuchung arbeitsschutzbezogener Lehrinhalte in Lehrbüchern metalltechnischer Ausbildungsberufe
}

menlehrplänen sind Aspekte des Arbeitsschutzes curricular verankert. Es stellt sich u. a. die Frage, inwieweit dieser curriculare Auftrag auch in der Praxis umgesetzt wird. Veröffentlichte Erkenntnisse hierzu konnten nicht identifiziert werden.

\section{Arbeitsschutzbezogene Kompe- tenzen von Auszubildenden}

Ziel beruflicher Bildungsprozesse ist die Entwicklung von Handlungskompetenz als „die Bereitschaft und Fähigkeit des Einzelnen, sich in beruflichen, gesellschaftlichen und privaten Situationen sachgerecht, durchdacht sowie individuell und sozial verantwortlich zu verhalten" [19]. Die genauere Charakterisierung beruflicher Kompetenzen erfolgt mittels berufsspezifischer Kompetenzmodelle. Diese beschreiben einerseits die verschiedenen Bereiche beruflicher Kompetenz und andererseits qualitative Unterschiede innerhalb der Inhaltsbereiche im Sinne hierarchisch geordneten Anforderungsniveaus [18]. Auf der Grundlage von Erkenntnissen aus dem Bereich der naturwissenschaftsdidaktischen Forschung sowie theoretisch-inhaltlichen Überlegungen ist anzunehmen, dass verschiedene Anforderungsniveaus über das Merkmal der Komplexität gegliedert in die Komplexitätsstufen Fakten, Zusammenhänge und
Konzept beschrieben werden können [7]. Bezüglich der Bereiche der beruflichen Kompetenz konnte in verschiedenen Studien $[8,11]$ gezeigt werden, dass diese in Bezug zu typischen beruflichen Anforderungssituationen sowie curricularen Schwerpunktsetzungen in der Berufsschule untergliedert werden kann [23]. Für den Beruf des Industriemechanikers sind dies z.B. die beruflichen Kompetenzbereiche physikalische und mechanische Grundlagen, Steuerungstechnik, Qualitäts- und Prüftechnik, Fertigung sowie Wartung/Instandhaltung/ Montage. Obwohl die Arbeitssicherheit bereits als zusätzlicher, relevanter Bereich der Kompetenz von gewerblichtechnischen Auszubildenden zugeordnet wurde [9], gibt es hierzu bisher weder weiterführende Konzeptualisierungen noch empirische Erkenntnisse.

Ziel des Abschnitts ist daher die Ableitung und Konkretisierung arbeitsschutzbezogener Kompetenzen auf Basis der Reflexion methodischer Grundlagen der Arbeitssicherheit. Das heißt, auf Basis der Methodik der Arbeitssicherheit [17] sowie der einschlägigen gesetzlichen Grundlagen werden im Folgenden die für den Bereich des Arbeitsschutzes relevanten beruflichen Anforderungssituationen bestimmt (• Abb. 1). Da diese Anforderungssituationen von spezifisch dafür ausgebildeten Personen (Nachweis der Fachkunde) bewältigt werden, wer- 


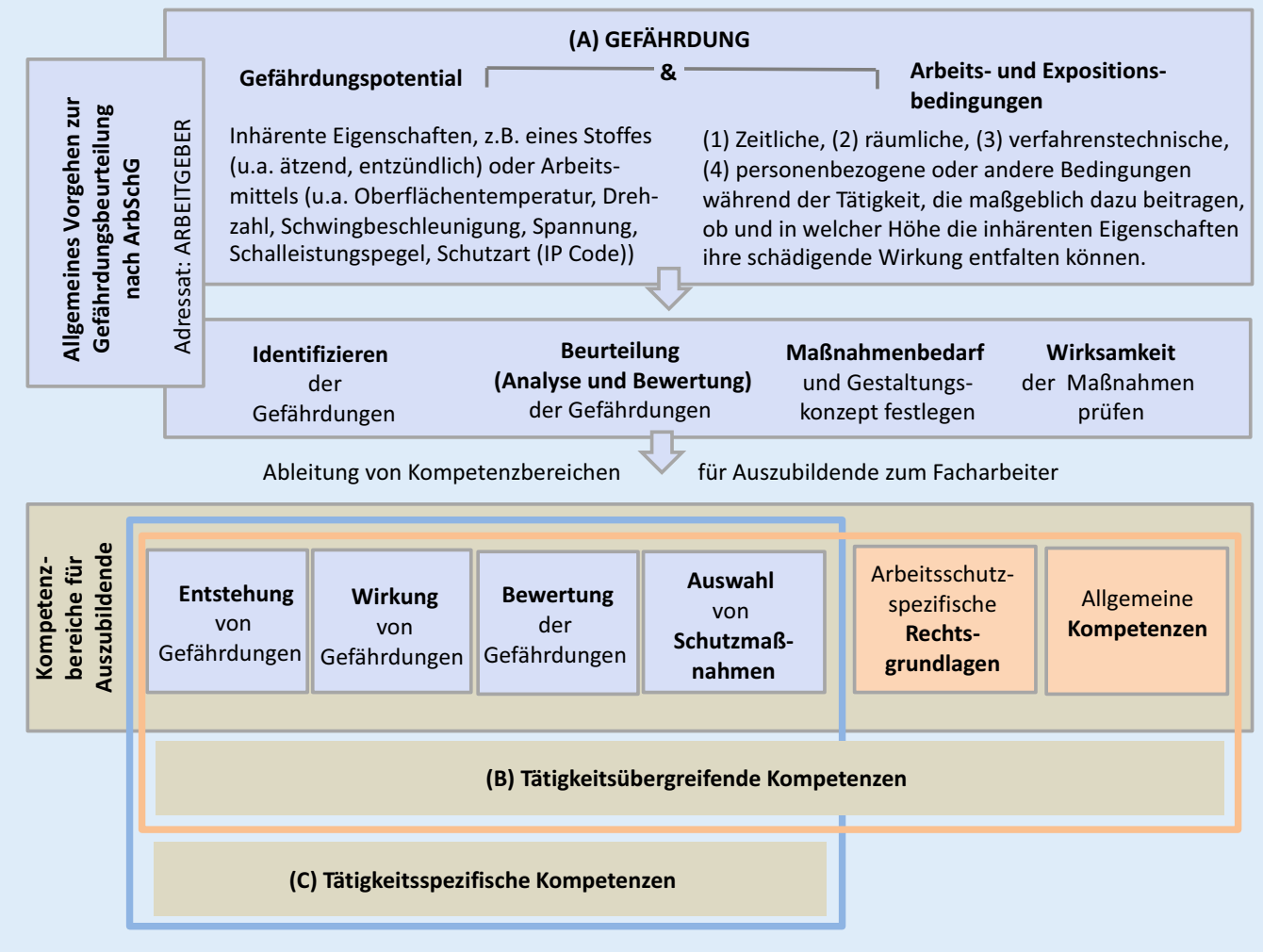

Abb. $1<$ Gefährdungsbegriff und arbeitsschutzspezifische Kompetenzbereiche für Auszubildende

den in einem zweiten Schritt in Bezug auf die Tätigkeiten der Auszubildenden (• Abb. 3) die für diese Beschäftigten relevanten Teilaspekte der Anforderungssituationen deduktiv abgeleitet (• Abb. 1) und beschrieben (• Abb. 2). Hintergrund ist, dass gem. $\$ 15$ Arbeitsschutzgesetz (ArbSchG) neben den fachkundigen Personen alle Beschäftigten gesetzlich verankerte Pflichten im Rahmen von Sicherheit und Gesundheitsschutz zu erfüllen haben.

Im Fokus des fachlich-methodischen Handlungsfeldes „Arbeitsschutz" stehen der Gefährdungsbegriff (• Abb. 1) sowie das Instrument der Gefährdungsbeurteilung. Der für das methodische Verständnis des betrieblichen Arbeitsschutzes zentrale Gefährdungsbegriff ist die Basis für die grundlegende Identifizierung und Beurteilung und damit auch für die Ermittlung des betrieblichen Schutzniveaus. Dabei wird das Ausmaß der Gefährdung über die beiden Bausteine „Gefährdungspotenzial“ und „Arbeits- und Expositionsbedingungen" beeinflusst und charakterisiert [17].
Mittels der Gefährdungsbeurteilung erfolgt die fachkundige Beurteilung der Gefährdungen. Ziel dieser Vorgehensweise ist die Feststellung, ob und - wenn notwendig - welcher Maßnahmenbedarf des Arbeitsschutzes bei der beurteilten Tätigkeit besteht. Ebenso ist festzustellen, wie dieser durch geeignete Schutzmaßnahmen realisiert werden kann. Welches Verfahren zur Gefährdungsbeurteilung im konkreten Anwendungsfall genutzt wird, ist u. a. abhängig vom Gefährdungsfaktor (z.B. Gefahrstoff oder Lärm), jedoch beinhalten alle am Markt befindlichen Verfahren grundsätzlich die nachfolgenden Schritte: Identifizierung der Gefährdung, Beurteilung der Gefährdung, Maßnahmenbedarf und Gestaltungskonzept festlegen sowie Wirksamkeit der Maßnahmen prüfen [17].

Obwohl die Schritte der Gefährdungsbeurteilung nicht im Tätigkeitsbereich des Facharbeiters liegen, bilden sie einen Orientierungspunkt für die sicherheitsbezogenen Kompetenzen des Facharbeiters, damit dieser befähigt wird, den gesetzlichen Pflichten der Beschäftigten nach ArbSchG gerecht werden $\mathrm{zu}$ können. Von diesem Orientierungspunk ausgehend, leiten sich aus den Schritten der Gefährdungsbeurteilung die sicherheitsbezogenen Kompetenzen ab (- Abb. 1).

Auf allgemeiner Ebene kann zusammengefasst werden, dass der kompetente Facharbeiter den im Rahmen der Gefährdungsbeurteilung festgelegten Maßnahmenbedarf verstehen und die auf seine Arbeitstätigkeit entfallenden Aspekte umsetzen sowie ggf. bei geänderten Bedingungen anpassen kann. Im Folgenden werden die Kompetenzbereiche, welche sich in Bezug zu den beruflichen Anforderungssituationen der fachkundigen Person für den Facharbeiter ergeben (-Abb. 1), detaillierter beschrieben (-Abb. 2).

Facharbeiter müssen die Entstehung von Gefährdungen sowie die sich ggf. bei Schadenseintritt ergebenden negativen Konsequenzen (Wirkung von Gefährdungen) nachvollziehen können, um ein Verständnis für den geeigneten Maßnahmenbedarf zu entwickeln.

Um diesen Maßnahmenbedarf situationsspezifisch anpassen $\mathrm{zu}$ können, müssen Facharbeiter zudem wirksame 
Zbl Arbeitsmed 2021 · 71:86-96 https://doi.org/10.1007/s40664-020-00414-6

(c) Der/die Autor(en) 2020

\section{A. Kahl · C. Frank · R. Erlebach}

\section{Eine kritische Untersuchung arbeitsschutzbezogener Lehrinhalte in Lehrbüchern metalltechnischer Ausbildungsberufe}

\section{Zusammenfassung}

Hintergrund. Ein Prädiktor für die Sicherheits-Compliance Beschäftigter ist das arbeitsschutzbezogene Wissen. Junge Beschäftigte sind überproportional von Arbeitsunfällen betroffen. Im Rahmen der beruflichen Ausbildung ist die Vermittlung von arbeitsschutzbezogenem Wissen und entsprechenden Kompetenzen ein wichtiges Ziel.

Ziel. Ziel der Studie ist zu untersuchen, für welche arbeitsschutzbezogenen Kompetenzen geeignete Inhalte in den Lehrbüchern für die gemeinsame Kernqualifikation metalltechnischer Ausbildungsberufe identifiziert werden können. Es wird somit der Frage nachgegangen, inwieweit der curriculare Auftrag, die berufsrelevanten
Aspekte des Arbeitsschutzes im Rahmen der Ausbildung zu vermitteln, in der Praxis auf Basis der zur Verfügung stehenden Lehrwerke umgesetzt werden kann.

Material und Methoden. Es wurden die in der beruflichen Grundausbildung der industriellen Metallberufe eingesetzten Lehrbücher $(N=6)$ auf Basis eines theoriebasiert entwickelten Kategoriensystems quantitativ und qualitativ analysiert.

Ergebnisse und Diskussion. Die Lehrbuchanalyse zeigt, dass in den ausgewählten Lehrbüchern die arbeitsschutzbezogenen Themenfelder stark eingeschränkt sind, da fast ausschließlich Inhalte im Bereich „Auswahl von Schutzmaßnahmen" thematisiert werden. Zudem werden in fast allen Lehrbüchern lediglich Tätigkeiten mit Gefahrstoffen sowie mit elektrischen Betriebsmitteln umfassender betrachtet. Die in den Lehrbüchern enthaltenen Schutzmaßnahmen stehen selten im Bezug zu den anderen arbeitsschutzbezogenen Kompetenzbereichen und weisen zudem häufig ein niedriges Komplexitätsniveau auf. Es besteht auf Grundlage der Lehrbuchinhalte kaum Potenzial, das für das Arbeitsschutzverständnis erforderliche „Denken in Zusammenhängen“ auszuprägen.

Schlüsselwörter

Lehrbuchanalyse - Arbeitsschutz - Arbeitssicherheit - Berufliche Bildung . Metalltechnik

\section{A critical study of occupational health and safety content in textbooks for metalworking training occupations}

\section{Abstract}

Background. A predictor of safety compliance among employees is occupational safety knowledge. Young employees are disproportionately affected by occupational accidents. Within the framework of vocational training, the imparting of occupational safety knowledge and corresponding competencies is an important goal.

Objective. The aim of study was to investigate for which occupational health and safety related competencies suitable contents can be identified in textbooks for metal engineering professions. The question was thus to what extent the curricular task of conveying the occupational health and safety aspects relevant to the workplace within the framework of training is also implemented in practice.

Material and methods. The textbooks $(N=6)$ used in the basic vocational training for industrial metalworking occupations were quantitatively and qualitatively analyzed based on a theory-based developed category system.

Results and discussion. The textbook analysis has shown that in the selected textbooks the included occupational health and safety-related subject areas are limited, as contents concerning the selection of protective measures are almost exclusively discussed. In addition, almost all textbooks only consider activities involving hazardous substances and electrical equipment more comprehensively from an occupational health and safety perspective. The protective measures contained in the textbooks are rarely related to the other areas of occupational health and safety competence and also often have a low level of complexity. Based on the textbook contents, there is hardly any potential to develop the thinking required for understanding occupational health and safety.

\section{Keywords}

Textbook analysis - Occupational health and safety - Occupational safety - Vocational education and training $\cdot$ Metal technology
Schutzmaßnahmen innerhalb des - in ihrem Tätigkeitskontext bestehenden Entscheidungsspielraums selbstständig auswählen können (Auswahl von Schutzmaßnahmen). Hierfür bedarf es nicht nur des Verständnisses von Gefährdungsentstehung und -wirkung, sondern darüber hinaus auch der Kenntnis über bestehende Bewertungsmaßstäbe und der grundlegenden Fähigkeit, diese für die Entscheidung über einen Maß- nahmenbedarf zu nutzen (Bewertung der Gefährdungen).

Basis dieser sicherheitsbezogenen Kompetenzbereiche ist ein Grundverständnis über den arbeitsschutzspezifischen Rechtsrahmen (arbeitsschutzspezifische Rechtsgrundlagen) sowie über allgemeine Grundsätze der Arbeitssicherheit und allgemeine Auswirkungen von Arbeitsunfällen und Berufskrankheiten (allgemeine Kompetenzen).
Die letzten beiden Kompetenzbereiche sind allgemeiner Natur und somit tätigkeitsübergreifend (• Abb. 1). Die weiteren Kompetenzbereiche umfassen neben den tätigkeitsübergreifenden auch tätigkeitsspezifische, d.h. auf die jeweilige Arbeitstätigkeit bezogene und somit berufsspezifische Kompetenzen. In - Abb. 2 sind die den sechs tätigkeitsübergreifenden Bereichen und vier tätigkeitsspezifischen Bereichen zugehörigen Kompetenzen beschrieben. Die rele- 


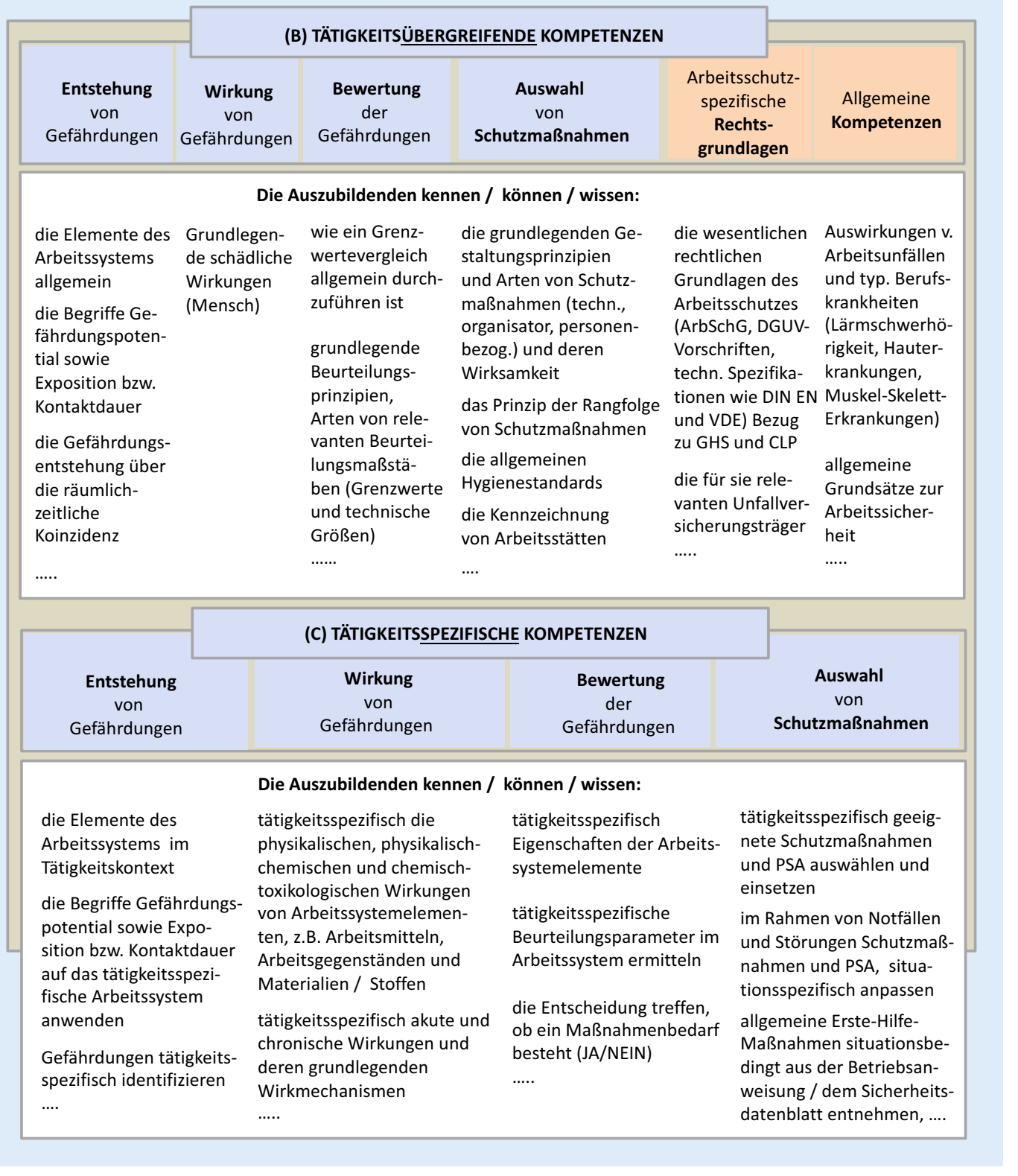

Abb. $2<$ Tätigkeitsübergreifende und -spezifische Kompetenzen im Arbeitsschutz für Auszubildende (Auszug, DGUV Deutsche Gesetzliche Unfallversicherung, GHS Globally Harmonized System, CLPClassification, Labelling and Packaging, PSA Persönliche Schutzausrüstung) vanten Tätigkeiten für die hier betrachteten industriellen Metallberufe sind in - Abb. 3 aufgeführt. Diese wurden auf Basis der Lernfelder 1 bis 4 der gemeinsamen Kernqualifikation der industriellen Metallberufe identifiziert und dann - in Abhängigkeit von den mit den Tätigkeiten verbundenen Gefährdungsaspekten - nach dem Prinzip der Ähnlichkeit geclustert.

\section{Ziel und Forschungsfragen der Studie}

Ziel der beruflichen Ausbildung ist, dass Auszubildende neben fachbezogenen
Kompetenzen auch arbeitsschutzbezogene Kompetenzen erwerben. Abgeleitet aus der Methodik des Arbeitsschutzes und den im Rahmen des Arbeitsschutzgesetzes formulierten Anforderungen an Beschäftigten bedeutet dies, dass Facharbeiter tätigkeitsübergreifende und tätigkeitsspezifische Kompetenzen in den Bereichen Entstehung von Gefährdungen, Wirkung von Gefährdungen, Beurteilung von Gefährdungen und Auswahl von Schutzmaßnahmen, allgemeine arbeitsschutzbezogene Kompetenzen sowie grundlegende Kenntnisse der relevanten Rechtsgrundlagen aufweisen sollten. Publikationen zum Thema
Arbeitsschutz und berufliche Bildung beschränken sich darauf, einerseits die Bedeutung des Arbeitsschutzes sowie die bisher unzureichende Implementation des Arbeitsschutzes im Rahmen der beruflichen Ausbildung zu betonen. Andererseits wird angemerkt, dass bisher kaum konzeptionelle Ansätze oder forschungsbasierte Erkenntnisse hierzu existieren [15, 20]. Zusammenfassend wird von allen Autoren eine Unzufriedenheit hinsichtlich der arbeitsschutzbezogenen Kompetenzen der Auszubildenden vermutet. Empirische Erkenntnisse hierzu liegen nicht vor. Um zunächst einen ersten Einblick darüber zu er- 
Abb. $3 \varangle$ Relevante Tätigkeiten für Auszubildende in industriellen Metallberufen halten, welche arbeitsschutzbezogenen Kompetenzen im berufstheoretischen Unterricht der gemeinsamen Kernqualifikation der industriellen Metallberufe potenziell erlernt werden können, werden im Rahmen dieser Studie Lehrbücher hinsichtlich der darin adressierten Arbeitsschutzinhalte analysiert. Schulbücher sind national und international für Lehrende ein wesentliches Hilfsmittel sowohl für die Unterrichtsvorbereitung als auch -durchführung $[4,25]$. Sie stellen somit einen maßgeblichen Mittler zwischen dem gesetzlich implementierten und dem praktisch realisierten Curriculum dar und ermöglichen durch ihre Funktion einen Zugang zu den tatsächlich im Unterricht thematisierten arbeitsschutzbezogenen Inhalten.

Auf Grundlage der Analyse der in der gemeinsamen Kernqualifikation metalltechnischer Ausbildungsberufe eingesetzten Lehrbücher soll daher folgenden Fragestellungen nachgegangen werden:

1. Welche tätigkeitsübergreifenden und tätigkeitsspezifischen Kompetenzen werden mit den in den Lehrbüchern thematisierten arbeitsschutzbezogenen Inhalten adressiert?

2. Unterscheiden sich die adressierten Kompetenzbereiche für die unterschiedlichen metalltechnischen Tätigkeiten?

\section{Methodik der Lehrbuchanalyse}

\section{Lehrbuchauswahl und Kategoriensystem}

Bei der Lehrbuchanalyse werden Lehrwerke berücksichtigt, welche im Rahmen der Ausbildung der industriellen Metallberufe zum Einsatz kommen und in NRW durch das Ministerium für Schule und Bildung (MSB NRW) zugelassen sind $[1,3,12,13,16,22]$. Die Lehrbücher sind nicht spezifisch für einzelne Bundesländer und werden daher auch deutschlandweit eingesetzt.

Für alle industriellen Metallberufe sind die Lernfelder 1 bis 4 Teil der gemeinsamen Kernqualifikation identisch. Zugleich wird in diesen ein Großteil der curricularen Anforderungen in Bezug auf den Bereich des Arbeitsschutzes thematisiert. Da in diesen Lernfeldern die curricularen Anforderungen bezogen auf den Arbeitsschutz sehr allgemein dargelegt werden und in den aufbauenden Lernfeldern der Fachstufe dagegen die arbeitsschutzbezogene Zielstellung in der Regel in den Kontext spezifischer Teilaspekte gesetzt wird, ist zudem davon auszugehen, dass methodische Grundlagen des Arbeitsschutzes im Rahmen der Kernqualifikation zu vermitteln sind. Aus diesen Gründen begrenzt sich die Lehrbuchanalyse auf die Lernfelder 1 bis 4 und somit auf die berufliche Grundbildung im ersten Lehrjahr. Die tätigkeitsspezifi- schen Kompetenzen werden daher auch lediglich für die in diesen Lernfeldern thematisierten Tätigkeiten (• Abb. 3) betrachtet.

Das Kategoriensystem leitet sich aus den $\mathrm{zu}$ vermittelnden arbeitsschutzbezogenen Kompetenzen ab. Entsprechend der Darstellung in Abschnitt „Arbeitsschutzbezogene Kompetenzen" ergibt sich das in Abb. 4 beschriebene, hierarchisch aufgebaute und nominal skalierte Kategoriensystem. Die Definitionen der einzelnen Kategorien können dem vorangestellten Theorieteil entnommen werden. Für die Kodierung wurden ausführliche Begriffsbestimmungen sowie die Kriterien für die einzelnen Kategorien in einem Kodierleitfaden ${ }^{1}$ detailliert beschrieben.

\section{Durchführung der Lehrbuch- analyse}

\section{Identifizierung und Auswahl der Analyseeinheiten}

Eine Analyseeinheit beschreibt die kleinste sachlogische Einheit, die eine Aussage zum Thema Arbeitsschutz in Form eines Fakts, eines Zusammenhangs oder eines Konzepts beinhaltet. In einem ersten Schritt wurden je Lehrwerk kapitelweise alle der Definition entsprechenden potenziellen Analyseeinheiten

\footnotetext{
1 Der Kodierleitfaden kann auf Nachfrage per E-Mail zugesendet werden.
} 


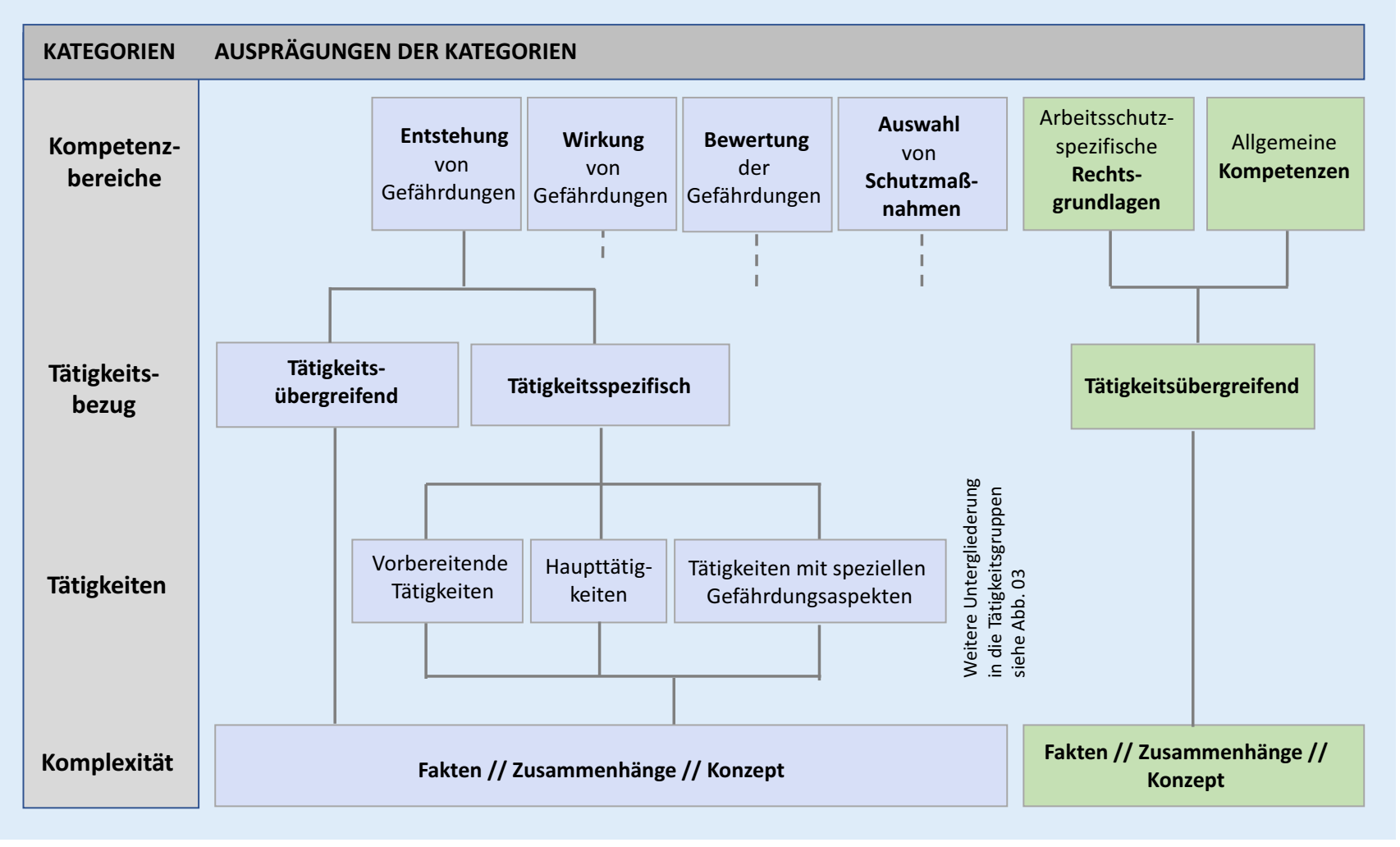

Abb. 4 \& Kategoriensystem

markiert. In einem zweiten Schritt wurden die markierten Analyseeinheiten entsprechend der nachfolgenden Kriterien geprüft und somit eine finale Festlegung der Analyseeinheiten vorgenommen.

Dabei wurden inhaltsgleiche Analyseeinheiten nur einmalig je Lehrbuch berücksichtigt. Dies gilt gleichermaßen, wenn der Inhalt sowohl grafisch als auch deskriptiv aufgeführt ist. Nicht berücksichtigt wurden illustrative Abbildungen und Aufgabenstellungen für die Auszubildenden, auch wenn diese einen inhaltlichen Bezug zum Arbeitsund Gesundheitsschutz aufweisen. $\mathrm{Zu}$ dem wurden Aussagen und Aspekte aufgenommen, welche fachlich zwar nicht primär arbeitsschutzspezifischen Charakter tragen, jedoch vor allem aus Gründen des Arbeits- und Gesundheitsschutzes in das Lehrwerk integriert wurden.

\section{Kategorisierung der Analyseeinheiten}

Um zu bestimmen, welcher Kategorienkombination eine Analyseeinheit zuzuordnen ist, wurden folgende Einzelentscheidungen hinsichtlich der vier zu berücksichtigenden Kategorien vorgenommen (• Abb. 4). Nachdem im ersten Schritt darüber entschieden wurde, in welchen Kompetenzbereich die Analyseeinheit einzuordnen ist, wurde im zweiten Schritt festgelegt, ob ein Tätigkeitsbezug vorliegt. Dabei wird in tätigkeitsspezifische und tätigkeitsübergreifende Analyseeinheiten unterschieden. Sofern ein Tätigkeitsbezug vorliegt, erfolgte im dritten Schritt eine Zuordnung der spezifischen Art der Tätigkeit (• Abb. 3). Im letzten Schritt wurde die Komplexitätsstufe bestimmt.

\section{Qualitative Gesamtbewertung der Analyseeinheiten}

Im Anschluss an die Zuordnung aller Analyseeinheiten eines Lehrbuchs gemäß (1) und (2) wurde geprüft, für welche der in - Abb. 2 beschriebenen arbeitsschutzbezogenen Kompetenzen fachspezifische Inhalte innerhalb des jeweiligen Lehrbuchs eingebunden sind und somit als Grundlage für die Gestaltung entsprechender Lehr- und Lernprozesse zur
Verfügung stehen. Als Ergebnis dieser Prüfung sind die mit den thematisierten Lehrbuchinhalten verbundenen Kompetenzen pro Lehrbuch dokumentiert worden.

\section{Gütekriterien}

In Anlehnung an das Vorgehen von [24] wurde das Kategoriensystem im diskursiven Austausch beider Wissenschaftlerinnen entwickelt, um so die beiden wissenschaftsdisziplinären Ansätze von Technikdidaktik und Sicherheitstechnik/ Arbeitssicherheit adäquat aufeinander $\mathrm{zu}$ beziehen. Diesem iterativen Ansatz entsprechend, wurden auf Basis einer ersten Version des Kategoriensystems die Lehrbücher kodiert und auftretende Unklarheiten und Mehrdeutigkeiten bei der Anwendung des Kategoriensystems sowie des Kodiervorgehens besprochen. Im Ergebnis wurde eine finale Version des Kategoriensystems erarbeitet.

Um die Güte des Kategoriensystems $\mathrm{zu}$ prüfen, wurden über alle Lehrbücher hinweg $20 \%$ aller Analyseeinheiten 


\section{Originalien}

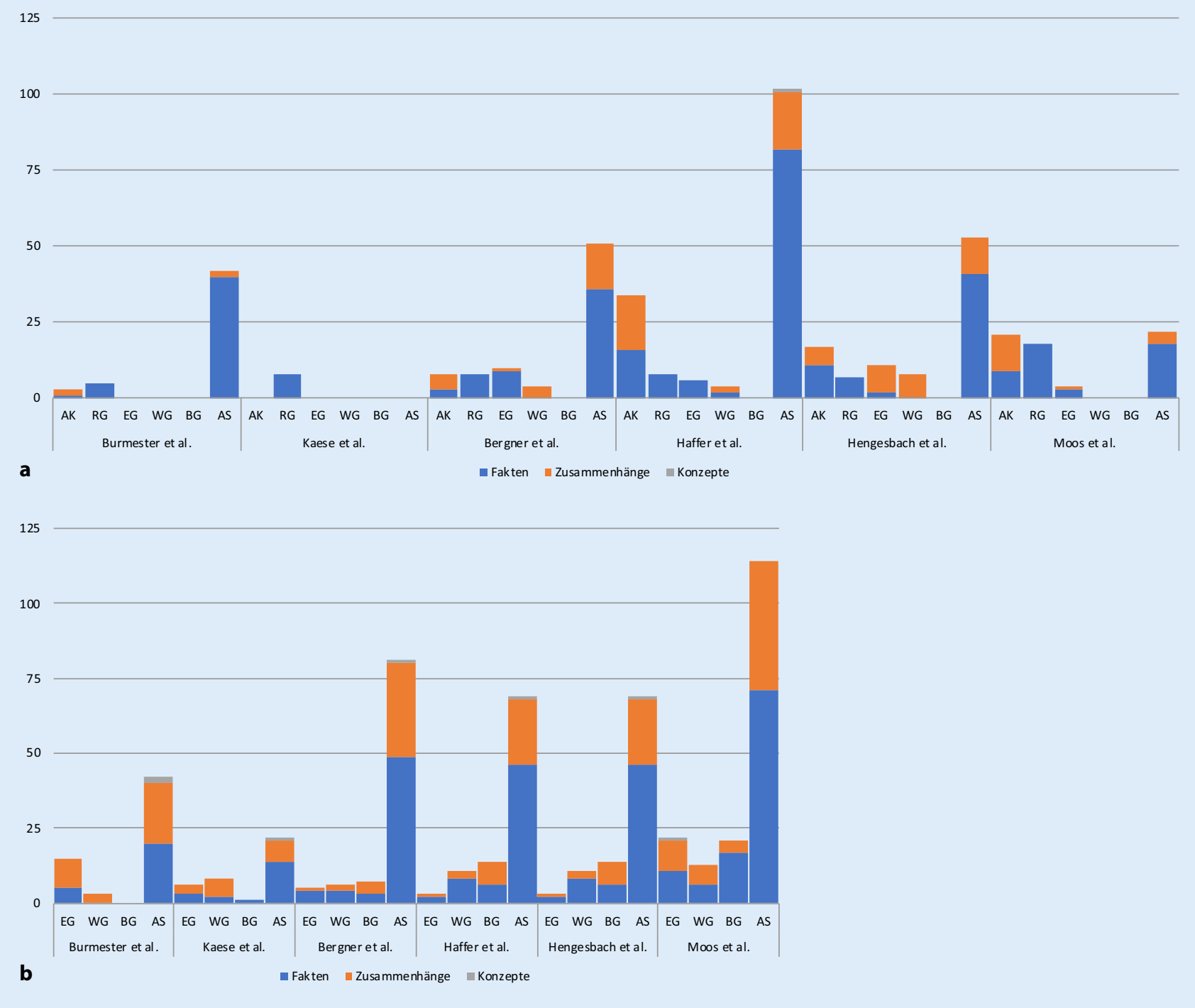

Abb. 5 \& Tätigkeitsübergreifende (a) und tätigkeitsspezifische (b) Kompetenzen in den untersuchten Lehrbüchern. AK Allgemeine Kompetenzen, RG Rechtsgrundlagen, EG Entstehung von Gefährdungen, WG Wirkung von Gefährdungen, $B G$ Beurteilung von Gefährdungen, AS Auswahl von Schutzmaßnahmen, Y-Achse = Anzahl der Analyseeinheiten

$\left(N_{G e s}=1.077^{2}, N_{20 \%}=216\right)$ jedes Lehrbuchs zufällig ausgewählt und durch einen zweiten externen Rater kodiert. Als Maß für die Interraterreliabilität wurde der Kappa-Koeffizient nach Cohen im Hinblick auf die beiden Forschungsfragen berechnet. Der berechnete Wert für die Kodierung des Tätigkeitsbezugs in Bezug auf die Kompetenzbereiche (Ebene 1 und 2 des Kategoriensystems in $\bullet$ Abb. 4) von $\kappa=0,62$ entspricht nach

2 Hauptautor (Gesamtzahl der Analyseeinheiten) $\rightarrow$ Burmester $\left(N_{\text {Ges }}=109\right)$, Kaese $\left(N_{G e s}=44\right)$, $\operatorname{Bergner}\left(N_{G e s}=182\right)$, Haffer $\left(N_{G e s}=260\right)$, Henges$\operatorname{bach}\left(N_{\text {Ges }}=245\right)$, $\operatorname{Moos}\left(N_{G e s}=237\right)$.
Landis und Koch [21] einer starken Übereinstimmung. Unter Einbezug der Komplexität (Ebene 4 mit den Ebenen 1 und 2 in - Abb. 4) ergibt sich immerhin noch ein $\kappa$-Wert von 0,55 , was einer moderaten Übereinstimmung entspricht. Angesichts der Komplexität des Kategoriensystems und des stark verzweigenden Entscheidungsbaums ist dies für die Beantwortung der Forschungsfrage, welche Kompetenzen mit den arbeitsschutzbezogenen Inhalten adressiert werden, ein akzeptables Ergebnis. Eine fast perfekte Übereinstimmung wurde zwischen den Ratern mit $\kappa=0,86$ bei der Beurteilung der Tätigkeiten in Bezug auf die
Kompetenzbereiche (Ebenen 1 und 3 in 0 Abb. 4) erzielt, was die zweite Forschungsfrage - nach den Unterschieden in den adressierten Kompetenzbereichen für die spezifischen metalltechnischen Tätigkeiten - betrifft.

\section{Ergebnisse}

Die Darstellung der Ergebnisse der Lehrbuchanalyse orientiert sich an der Unterteilung der arbeitsschutzbezogenen Kompetenzen in tätigkeitsübergreifende und tätigkeitsspezifische Kompetenzen. Neben der quantitativen Betrachtung (Anzahl von Analyseeinheiten je Kom- 

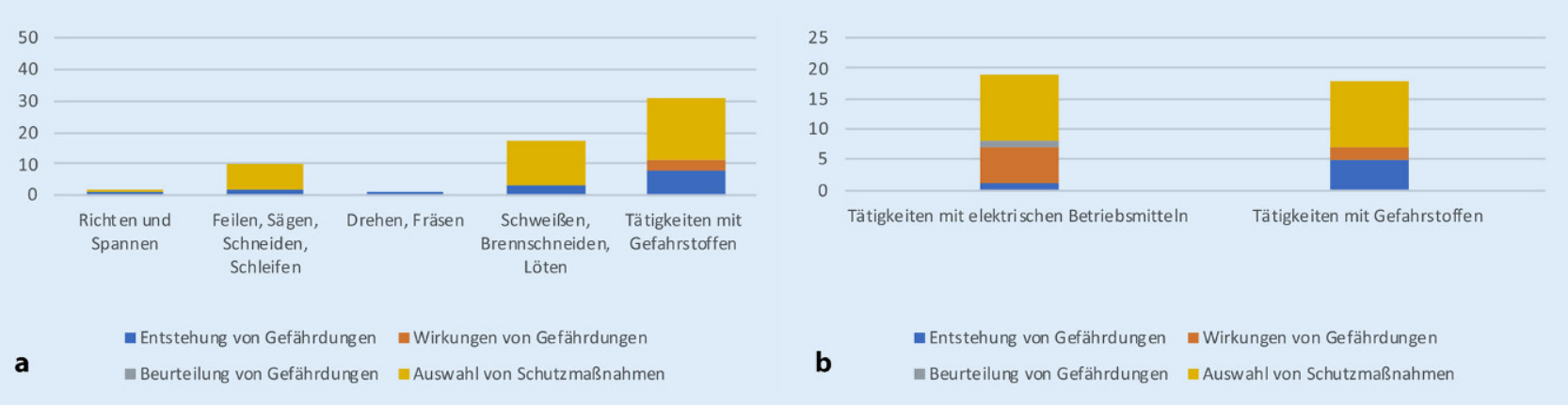

a Entstehung von Gefährdungen $\quad$ Wirkungen von Gefährdungen
a Beurteilung von Gefährdungen $\quad$ Auswahl von Schutzmaßnahmen
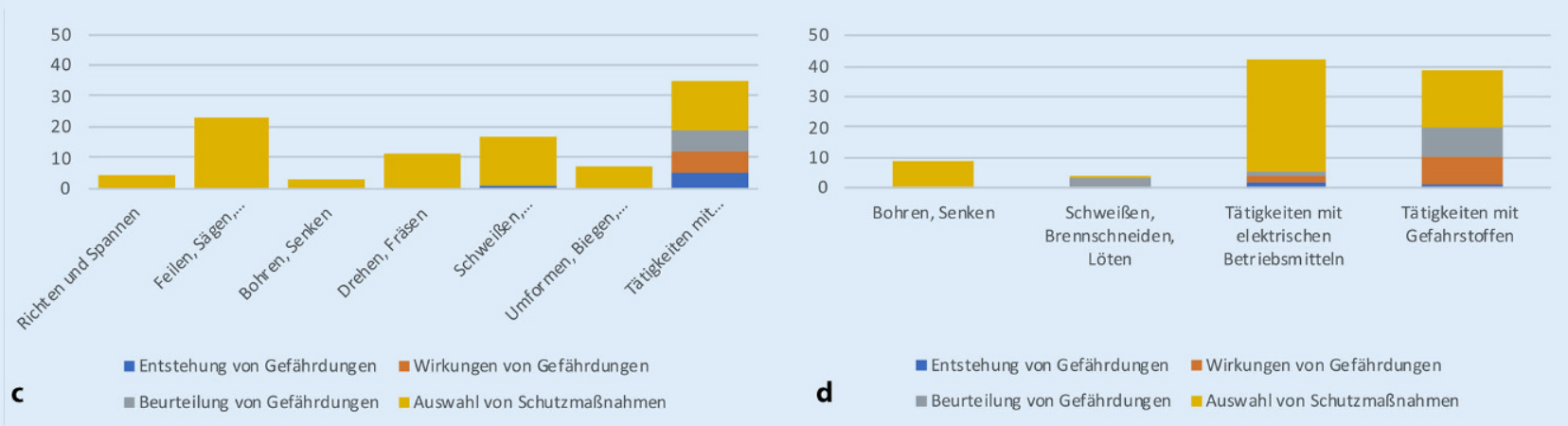

m Beurteilung von Gefährdungen $\approx$ Auswahl von Schutzmaßnahmen
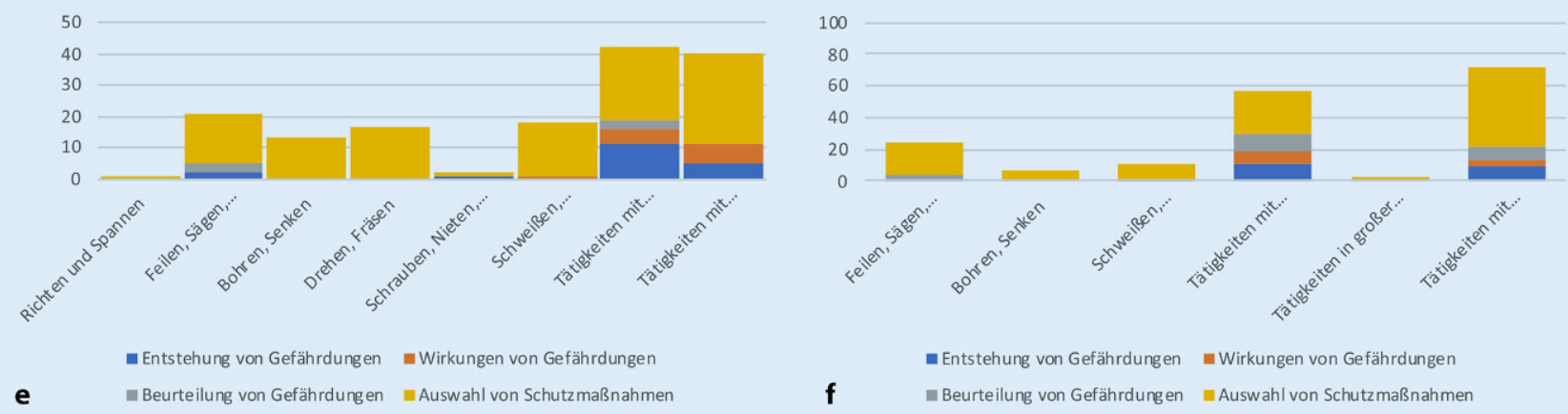

Abb. 6 ॥ Tätigkeitsspezifische Kompetenzen in den untersuchten Lehrbüchern nach Tätigkeitsbereichen. a Lehrbuch 1 (Burmester et al. [3]), b Lehrbuch 2 (Kaese et al. [16]), c Lehrbuch 3 (Bergner et al. [1]), d Lehrbuch 4 (Haffer et al. [12]), e Lehrbuch 5 (Hengesbach et al. [13]), f Lehrbuch 4 (Moos et al. [22])

petenzbereich) wurde für alle Kompetenzbereiche eine inhaltliche Analyse auf Basis der beschriebenen Kompetenzen (• Abb. 2) vorgenommen. Die quantitative Auswertung der Verteilung der Analyseeinheiten über die verschiedenen Kompetenzbereiche je Lehrbuch ist in Abb. 5 zusammenfassend dargestellt.

\section{Befunde zu tätigkeitsüber- greifenden Kompetenzen}

Für die tätigkeitsübergreifenden Kompetenzen zeigt sich abgesehen von einer Ausnahme in allen untersuchten Lehr- büchern, dass der überwiegende Teil der Analyseeinheiten dem Kompetenzbereich "Auswahl von Schutzmaßnahmen“ zuzurechnen ist. Für die weiteren Kompetenzbereiche ist hingegen festzustellen, dass nur wenige arbeitsschutzspezifische Aussagen getroffen werden und somit nur vereinzelt Kompetenzen in diesen Bereichen aufgreifen. Zudem liegt für diese Kompetenzbereiche keine lehrbuchübergreifend einheitliche Verteilung vor.

Hinsichtlich der Komplexität der Analyseeinheiten kann konstatiert werden, dass $73,4 \%$ der Komplexitätsstufe
Fakten und 26,4\% der Komplexitätsstufe Zusammenhänge zuzuordnen sind. Lediglich eine der 454 tätigkeitsübergreifenden Analyseeinheiten entfällt auf die Komplexitätsstufe Konzept. Die Verteilung der Komplexitätsstufen Fakten und Zusammenhänge ist je Kompetenzbereich und Lehrwerk sehr unterschiedlich (• Abb. 5).

\section{Befunde zu tätigkeitsspezifischen Kompetenzen}

Im Rahmen der Identifizierung der tätigkeitsspezifischen Analyseeinheiten stan- 
den die in den Lehrbüchern gemäß LF 1 bis 4 eingebundenen metalltechnischen Tätigkeiten im Fokus (• Abb. 3.). Diese wurden in entsprechende Tätigkeitsgruppen mit ähnlichem Ausmaß und Charakter der potenziellen Gefährdungen eingeteilt, z. B. die Tätigkeitsgruppe „Schrauben, Nieten, Hämmern“.

Hinsichtlich der tätigkeitsspezifischen Kompetenzen im Arbeitsschutz zeichnet sich ebenfalls ein diverses Bild ab. Über die Gesamtheit der Tätigkeitsbereiche hinweg (• Abb.5) lässt sich grundsätzlich zusammenfassen: In allen Lehrbüchern sind deutlich mehr als jeweils 50\% aller identifizierten Analyseeinheiten dem Kompetenzbereich „Auswahl von Schutzmaßnahmen“ zuzurechnen.

Die weiteren Analyseeinheiten verteilen sich auf die übrigen Kompetenzbereiche, wobei sich auch hierfür lehrbuchübergreifend kein homogenes Verteilungsbild ergibt. Daher wird die nachfolgende Betrachtung der Ergebnisse auf Ebene der spezifischen Tätigkeitsbereiche vorgenommen (•Abb.6).

Alle Lehrbücher verweisen auf den Tätigkeitsbereich „Tätigkeiten mit Gefahrstoffen“. Der Tätigkeitsbereich „Tätigkeiten mit elektrischen Betriebsmitteln" wird in 4 von 6 Lehrbüchern im arbeitsschutzspezifischen Kontext aufgegriffen. Gleiches gilt für die Tätigkeitsbereiche „Feilen, Sägen, Schneiden, Schleifen“ sowie „Bohren und Senken“.

Ein ganzheitliches arbeitsschutzbezogenes Denken und Handeln erfordert, dass alle Kompetenzbereiche (von der Entstehung, über die Wirkung und die Beurteilung der Gefährdungen bis hin zur Maßnahmenauswahl) thematisiert und methodisch verknüpft werden. Lehrbuchübergreifend werden verschiedene Kompetenzbereiche jedoch nur für die "Tätigkeiten mit Gefahrstoffen“ sowie „Tätigkeiten mit elektrischen Betriebsmitteln“ adressiert. Für die weiteren Tätigkeitsbereiche wird in der Regel nur der Kompetenzbereich "Auswahl von Schutzmaßnahmen" aufgeführt.

Die in den LF 1 bis 4 vorgegebene Breite an Tätigkeiten wird im arbeitsschutzspezifischen Kontext nur von wenigen Lehrbüchern aufgegriffen $[1,13,22]$. Dennoch gilt auch für diese Lehrbücher, dass für den Großteil von Tätigkeits- bereichen lediglich die "Auswahl von Schutzmaßnahmen" thematisiert wird.

Hinsichtlich der Komplexität der Analyseeinheiten zeigt sich lehrbuchübergreifend, dass $61,3 \%$ der Komplexitätsstufe Fakten und $37,4 \%$ der Komplexitätsstufe Zusammenhänge zuzuordnen sind. Lediglich 1,3\% der 612 tätigkeitsspezifischen Analyseeinheiten entfallen auf die Komplexitätsstufe Konzept (• Abb. 5). Im Vergleich zu den tätigkeitsübergreifenden Kompetenzbereichen sind die Analyseeinheiten der tätigkeitsspezifischen Kompetenzbereiche im Durchschnitt auf einer höheren Komplexitätsstufe.

\section{Diskussion}

Arbeitsschutzbezogene Kompetenzen stellen einen Teil der von Auszubildenden zu erwerbenden beruflichen Kompetenzen dar. In Anbetracht der Tatsache, dass insbesondere junge Beschäftigte überproportional von $\mathrm{Ar}$ beitsunfällen betroffen sind [6], stellt sich u.a. die Frage, inwieweit der curriculare Auftrag, die berufsrelevanten Aspekte des Arbeitsschutzes im Rahmen der Ausbildung zu vermitteln, auch in der Praxis umgesetzt wird. Aktuell liegen hierzu noch keine Erkenntnisse vor. Da Lehrbücher ein zentrales Hilfsmittel für die Unterrichtsvorbereitung darstellen, sind sie ein guter Indikator für das praktisch realisierte Curriculum. Im Rahmen der vorliegenden Studie wurden in einem ersten Schritt die in der gemeinsamen Kernqualifikation der industriellen Metallberufe verwendeten Lehrbücher analysiert. Hierfür wurden die in den Lehrbüchern enthaltenen arbeitsschutzspezifischen Inhalte den im Rahmen der Ausbildung zu vermittelnden arbeitsschutzbezogenen tätigkeitsübergreifenden und -spezifischen Kompetenzen zugeordnet.

Die Analyse der tätigkeitsübergreifenden Kompetenzen hat gezeigt, dass der Großteil der Analyseeinheiten dem Bereich „Auswahl von Schutzmaßnahmen" zuzuordnen ist und alle weiteren Kompetenzbereiche (Entstehung, Wirkung oder auch Bewertung von Gefährdungen) kaum in den Lehrwerken berücksichtigt werden.
Weiterhin ist festzustellen, dass nahezu drei Viertel aller Analyseeinheiten lediglich die Aufzählung arbeitsschutzbezogener Fakten darstellen und somit von geringer Komplexität sind. Der Kompetenzbereich „Auswahl von Schutzmaßnahmen" beschreibt den letzten Schritt des methodischen Vorgehens im Arbeitsschutz, d.h. dieser Schritt baut in der Sachlogik auf die vorherigen Schritte (Entstehung von Gefährdungen, Wirkung von Gefährdungen und Bewertung von Gefährdungen) auf und kann deswegen nicht singulär betrachtet werden. Da die in den Lehrbüchern enthaltenen Schutzmaßnahmen kaum in Bezug zu den anderen arbeitsschutzbezogenen Kompetenzbereichen gestellt werden und zudem häufig ein niedriges Komplexitätsniveau vorliegt, besteht auf Grundlage der Lehrbuchinhalte wenig Potenzial, das für das Arbeitsschutzverständnis erforderliche „Denken in Zusammenhängen" auszuprägen. Aufgrund der curricularen Struktur der Lehrpläne ist vor allem ein grundlegendes Arbeitsschutzverständnis im Rahmen der Kernqualifikation zu vermitteln, damit dieses in den darauf aufbauenden Lernfeldern auf weitere Tätigkeiten übertragen werden kann.

Es wäre für den Bereich der übergreifenden arbeitsschutzbezogenen Themenfelder notwendig, dass Anliegen, Zielstellung und Vorgehen des Arbeitsschutzes in einem fachlichen Kontext erläutert werden. Dazu zählt vor allem die Gefährdungsbeurteilung als das zentrale Instrument des Arbeitsschutzes. In keinem einzigen der untersuchten Lehrwerke wird auf dieses Instrument verwiesen. Dies ist deshalb besonders kritisch zu bewerten, da Auszubildende und Beschäftigte Adressaten der Gefährdungsbeurteilung sind und deren Sicherheit sowie die Sicherheit Dritter auf der Ableitung geeigneter Maßnahmen im Rahmen der Gefährdungsbeurteilung beruht. Eine rechtliche Einordnung des Arbeitsschutzes im Sinne einer Klarstellung der Rechtsverbindlichkeit sowie der bestehenden Aufgaben und Pflichten des Arbeitgebers einerseits sowie der Beschäftigten andererseits findet in keinem der untersuchten Lehrbücher statt. Darüber hinaus muss kritisch angemerkt werden, 
dass der in den Lehrwerken priorisierte Blick auf Arbeitsunfälle die betriebliche Realität nur sehr eingeschränkt widerspiegelt.

Die trotz verschiedener Kampagnen der deutschen gesetzlichen Unfallversicherung (DGUV) unverändert hohe Zahl an Berufserkrankungen (u.a. jährliche Neuerkrankungen) macht ferner deutlich, dass gerade auch in der beruflichen Ausbildung ein Schwerpunkt auf diese latenzzeitbezogenen arbeitsbedingten Erkrankungen gelegt werden sollte. Die untersuchten Lehrbücher bieten kaum Informationen, die es ermöglichen, diese Thematik im Unterricht aufzugreifen.

Die starke Priorisierung des Kompetenzbereichs „Auswahl von Schutzmaßnahmen" zeigt sich auch für die tätigkeitsspezifischen Kompetenzen. Ausnahmen hiervon stellen die Tätigkeiten mit Gefahrstoffen und mit elektrischen Betriebsmitteln dar. Dass insbesondere für diese Bereiche Aussagen zu allen bzw. fast allen tätigkeitsspezifischen Kompetenzen vorliegen, zeigt, dass Konsens über die Bedeutsamkeit dieser Gefährdungsfaktoren besteht.

Als Fazit lässt sich konstatieren, dass der methodische Ansatz des Arbeitsschutzes in allen untersuchten Lehrbüchern nicht ausreichend widergespiegelt wird.

Die festgestellte Verengung des Themas Arbeitsschutz auf eine vom methodischen Konzept losgelöste Forderung nach Schutzmaßnahmen kann zur Konsequenz haben, dass es nur in wenigen Fällen möglich ist, den Zusammenhang zwischen der Entstehung einer Gefährdung und den geeigneten Schutzmaßnahmen eigenständig abzuleiten und ganzheitlich zu verstehen. Diese Form der Darstellung in den Lehrbüchern ermöglicht somit nur ungenügend, dass die Lernenden ein Verständnis für den Arbeitsschutz und damit auch für die eigene Sicherheit entwickeln können. Es bedarf jedoch dieses Verständnisses, um die Entwicklung einer zeitgemäßen, betrieblichen Sicherheitskultur zu fördern. Wenn Beschäftigte nicht erklären können, warum sie sich mit Sicherheitsfragen im Rahmen ihrer Tätigkeit gezielt auseinandersetzen müssen und weshalb geeignete Schutzmaßnahmen notwen- digerweise zu ergreifen sind, stellt der betriebliche Arbeitsschutz für sie wahrscheinlich nur einen rechtsformalen Akt ohne Mehrwert dar.

Die untersuchten Lehrbücher bieten somit keine ausreichende fachliche und methodische Grundlage, um die Kompetenzen zur Erfüllung der im Arbeitsschutzgesetz festgelegten Pflichten zur Mitwirkung, zum Eigenschutz sowie zum Schutzes Dritter im Rahmen des berufstheoretischen Unterrichts $\mathrm{zu}$ vermitteln.

Inwieweit diese Kompetenzen durch die Lehrenden tatsächlich vermittelt werden, kann auf Basis der vorliegenden Lehrbuchanalyse nicht beantwortet werden. Hierfür sind weiterführende Untersuchungen notwendig.

\section{Fazit für die Praxis}

- Facharbeiter in der Metalltechnik müssen im Rahmen ihrer Ausbildung arbeitsschutzbezogene Kompetenzen in den Bereichen Entstehung, Wirkung und Bewertung der Gefährdungen sowie Auswahl von Schutzmaßnahmen erwerben.

- In den in der Berufsschule eingesetzten Lehrbüchern finden sich fast ausschließlich Inhalte zum Bereich „Auswahl von Schutzmaßnahmen“.

- In fast allen Lehrbüchern werden lediglich Tätigkeiten mit Gefahrstoffen sowie mit elektrischen Betriebsmitteln umfassender aus der Sicht des Arbeitsschutzes betrachtet.

- Die in den Lehrbüchern enthaltenen Schutzmaßnahmen stehen zu selten im Bezug zu den anderen arbeitsschutzbezogenen Kompetenzbereichen und weisen zudem häufig ein niedriges Komplexitätsniveau auf.

- Es besteht kaum Potenzial, auf Grundlage der Lehrbuchinhalte das für das Arbeitsschutzverständnis erforderliche "Denken in Zusammenhängen" auszuprägen.

\section{Korrespondenzadresse}

Prof. Dr.-Ing. habil. Anke Kahl

Lehrstuhl für Sicherheitstechnik/

Arbeitssicherheit, Fakultät Maschinen-

bau/Sicherheitstechnik, Bergische Universität Wuppertal

Gaußstraße 20, 42119 Wuppertal, Deutschland akahl@uni-wuppertal.de

Funding. Open Access funding enabled and organized by Projekt DEAL.

\section{Einhaltung ethischer Richtlinien}

Interessenkonflikt. A. Kahl, C. Frank und R. Erlebach geben an, dass kein Interessenkonflikt besteht.

Für diesen Beitrag wurden von den Autoren keine Studien an Menschen oder Tieren durchgeführt. Für die aufgeführten Studien gelten die jeweils dort angegebenen ethischen Richtlinien.

Open Access. Dieser Artikel wird unter der Creative Commons Namensnennung 4.0 International Lizenz veröffentlicht, welche die Nutzung, Vervielfältigung, Bearbeitung, Verbreitung und Wiedergabe in jeglichem Medium und Format erlaubt, sofern Sie den/die ursprünglichen Autor(en) und die Quelle ordnungsgemäß nennen, einen Link zur Creative Commons Lizenz beifügen und angeben, ob Änderungen vorgenommen wurden.

Die in diesem Artikel enthaltenen Bilder und sonstiges Drittmaterial unterliegen ebenfalls der genannten Creative Commons Lizenz, sofern sich aus der Abbildungslegende nichts anderes ergibt. Sofern das betreffende Material nicht unter der genannten Creative Commons Lizenz steht und die betreffende Handlung nicht nach gesetzlichen Vorschriften erlaubt ist, ist für die oben aufgeführten Weiterverwendungen des Materials die Einwilligung des jeweiligen Rechteinhabers einzuholen.

Weitere Details zur Lizenz entnehmen Sie bitte der Lizenzinformation auf http://creativecommons.org/ licenses/by/4.0/deed.de.

\section{Literatur}

1. Bergner O, Fehrmann M, Hillebrand T et al (2017) Metallbau- und Fertigungstechnik, 11. erweiterte Auflage. Aufl. Verlag Europa-Lehrmittel, Haan

2. Burke MJ, Salvador RO, Smith-Crowe K, ChanSerafin S, Smith A, Sonesh S (2011) The dread factor: How hazards and safety training influence learning and performance. J Appl Psychol 96(1):46-70

3. Burmester J, Dillinger J, Escherich $\mathrm{W}$ et al (2017) Fachkunde Metall, 58., neu bearbeitete Auflage. Aufl. Verlag Europa-Lehrmittel, Nourney, Vollmer \& Co. KG, Haan

4. Chen J-J (2002) Reforming textbooks, reshaping school knowledge:Taiwan's textbookderegulation in the 1990s1. Pedagogy Cult Soc 10(1):39-72

5. Christian MS, Bradley JC, Wallace JC, Burke MJ (2009) Workplace safety: a meta-analysis of the 
roles of person and situation factors. J Appl Psychol 94(5):1103-1127

6. DGUV (2018) Arbeitsunfallgeschehen 2017. https://publikationen.dguv.de/zahlen-fakten/ schwerpunkt-themen/3479/arbeitsunfallgesch ehen-2017.Zugegriffen:30. Okt. 2020

7. Frank C, Bernholt S, Parchmann I (2016) Modellierung des Zusammenhangs allgemeiner und beruflicher Kompetenzen für die Domäne Chemie. ZDidak Naturwiss 22(1):43-60

8. Geißel B (2008) Ein Kompetenzmodell für die elektrotechnische Grundbildung. Kriteriumsorientierte Interpretation von Leistungsdaten. In: Nickolaus R, Schanz H (Hrsg) Didaktik der gewerblich-technischen Berufsbildung: konzeptionelle Entwürfe und empirische Befunde. Diskussion Berufsbildung. Schneider Verlag Hohengehren, , S 121-142

9. Gönnenwein A, Nitzschke A, Schnitzler A (2011) Fachkompetenzerfassung in der gewerblichen Ausbildung am Beispiel des Ausbildungsberufs Mechatroniker/-in. Entwicklung psychometrischer Fachtests. Berufsbild Wiss Prax 5:14-18

10. Griffin MA, Neal A (2000) Perceptions of safety at work: a framework for linking safety climate to safety performance, knowledge, and motivation. JOccup Health Psychol 5(3):347-368

11. Gschwendtner T, Abele S, Nickolaus R (2009) Computersimulierte Arbeitsproben: Eine Validierungsstudie am Beispiel der Fehlerdiagnoseleistungen von KfZ-Mechatronikern. Z Berufs-Wirtsch 105(4):557-578

12. Haffer R, Becker-Kavan A, van den Boom $G$ et al (2014) Grundkenntnisse Industrielle Metallberufe. Lernfelder 1-4, 6. aktualisierte Auflage. Aufl. Handwerk und Technik,

13. Hengesbach K, Koch F, Pyzalla G et al (2016) Fachwissen Metall: Grundstufe und Fachstufe 1 Schülerband, 11. Aufl. Bildungsverlag EINS, Köln

14. Jiang L, Yu G, Li Y, Li F (2010) Perceived colleagues' safety knowledge/behavior and safety performance: safetyclimate as a moderatorin a multilevel study. Accid Anal Prev 42(5):1468-1476

15. Kaeding A (2011) Arbeitsschutz in der Berufsausbildung. Chancen und Wettbewerbsvorteile. VS, Wiesbaden

16. Kaese J, Langanke L, Schmid K-G, Sokele G, Tiedt G (2016) Metalltechnik. Grundwissen Lernfelder 1-4, 4. Aufl. Westermann, Braunschweig

17. Kahl A (2019) Fachliche Grundlagen der Arbeitssicherheit. Erich-Schmidt-Verlag, Berlin

18. Klieme E, Maag-Merki K, Hartig J (2007) Kompetenzbegriff und Bedeutung von Kompetenzen im Bildungswesen. In: Hartig J, Klieme E (Hrsg) Möglichkeiten und Voraussetzungen technologiebasierter Kompetenzdiagnostik. Bundesministerium für Bildung und Forschung, Bonn, S5-15

19. KMK (1991) Rahmenvereinbarung über die Berufsschule. Beschluss der Kultusministerkonferenz vom 14./15. März 1991

20. Krüger W (1983) Arbeitssicherheit als berufspaedagogisches Problem. Wirtschaftsverlag NW,

21. Landis JR, Koch GG (1977) The measurement of observer agreement for categorical data. Biometrics. https://doi.org/10.2307/2529310

22. Moos J, Wagenleiter HW, Wollinger P (2015) Grundkenntnisse Metallbauer und Konstruktionsmechaniker. Lernfelder 1-4, 4., überarbeitete Auflage. Aufl. Handwerk und Technik,

23. Nickolaus R, Lazar A, Norwig K (2012) Assessing professional competences and their development in vocational education in Germany: state of research and perspectives. making it tangible. In: Bernholt S, Neumann K, Norwig K (Hrsg) Learning outcomes in science education. Waxmann, Münster, S129-150

24. Slough SW, McTigue EM, Kim S, Jennings SK (2010) Science textbooks' use of graphical representation: a descriptive analysis of four sixth grade science texts. Read Psychol 31(3):301-325

25. Valverde GA, Bianchi LJ, Wolfe RG, Schmidt WH, Houang RT (2002) According to the book: Using TIMSS to investigate the translation of policy into practice through the world of textbooks. Springer Science \& Business Media, 